\title{
Can back shape screening be used to predict the risk of falls in the elderly? An exploratory study investigating the relationship between spinal curvature and postural sway in healthy subjects C Johnson*, JA Bettany-Saltikov and JG Warren
}

\author{
Address: University of Teesside, School of Health and Social Care Borough Road, Middlesbrough, TS1 3BA, UK \\ Email: C Johnson* - j.b.saltikov@tees.ac.uk \\ * Corresponding author
}

from 6th International Conference on Conservative Management of Spinal Deformities Lyon, France. 21-23 May 2009

Published: 14 December 2009

Scoliosis 2009, 4(Suppl 2):O25 doi:10.1 186/1748-716I-4-S2-O25

This abstract is available from: http://www.scoliosisjournal.com/content/4/S2/O25

(C) 2009 Johnson et al; licensee BioMed Central Ltd.

\section{Objectives}

The primary objective of this study was to evaluate the relationship between spinal curvature and postural sway in the frontal and sagittal planes in asymptomatic young adults. A secondary objective was to look at this relationship over a time period of 15 minutes.

\section{Background}

One in three people over the age of 65 years experiences a fall every year in the UK. A significant factor contributing to the higher risk of falling in the elderly is attributed to an increase in postural sway which is a good indicator of static standing balance. Alterations in back shape with age have also been linked to an increase in postural sway. There is however a scarcity of research that has investigated this relationship. It may be possible to predict those at risk of falling through back shape screening, thereby preventing accidents before they occur.

\section{Methods}

Twenty five healthy young adults, ages 20 to 32 years volunteered to take part in this study. Approval was gained from the University of Teesside, Ethics committee. A Microscribe 3DX Digitizer was used to measure spinal curvature and a Kistler Force Plate calculated postural sway values. Each participant stood on the force plate for a period of 15 minutes. Back shape measurements and a 30 second force plate reading were taken simultaneously at the start and again at 15 minutes.

\section{Results}

A significant positive correlation was found between Lumbar Lordosis and Anteroposterior Sway measured at the start $(\mathrm{r}=0.398, \mathrm{p}<0.05)$. The change in Mediolateral sway over 15 minutes was also significantly different $(\mathrm{p}<$ 0.05). Further, general trends demonstrated that increasing spinal angles in the sagittal plane correlated with increasing anteroposterior sway while increasing spinal angles in the frontal plane correlated with increasing mediolateral sway.

\section{Conclusion}

The research demonstrated the possibility of identifying those at risk of falling, from back shape and postural sway values. Those at risk could then be referred to essential 'balance and falls' rehabilitation classes. The authors acknowledge the limitations of this study, and that it was conducted on normal healthy young subjects. A larger sample on elderly patients is required to further evaluate these preliminary results. 